\title{
EFEITO DE INSETICIDAS USADOS NA CULTURA DO MILHO (Zea mays L.) SOBRE NINFAS E ADULTOS DE Doru luteipes (SCUDDER) (DERMAPTERA: FORFICULIDAE) EM SEMICAMPO
}

\author{
ANA CAROLINA REDOAN ${ }^{1}$, GERALDO ANDRADE CARVALHO ${ }^{1}$, IVAN CRUZ ${ }^{2}$
} MARIA DE LOURDES CORRÊA FIGUEIREDO ${ }^{2}$ e RAFAEL BRAGA DA SILVA ${ }^{3}$

${ }^{1}$ Mestre em Agronomia/Entomologia, Departamento de Entomologia UFLA, Cx. Postal 3037, CEP: 37200-000, Lavras, MG, Brasil,E-mail: ac.redoan@gmail.com

'Professor Doutor do Departamento de Entomologia UFLA, Cx. Postal 3037, CEP: 37200-000, Lavras, MG, Brasil, E-mail: gacarval@den.ufla.br

${ }^{2}$ Embrapa Milho e Sorgo, CEP: 35701-970, Sete Lagoas, MG, Brasil,E-mail: ivancruz@cnpms. embrapa.br; figueiredomlc@yahoo.com.br

${ }^{3}$ Doutorando em Ecologia e Recursos Naturais, Departamento de Ecologia e Biologia Evolutiva UFSCar, CEP: 13565-905, São Carlos, SP, Brasil,E-mail: rafaelentomologia@yahoo.com.br

Revista Brasileira de Milho e Sorgo, v.9, n.3, p. 223-235, 2010

RESUMO - Doru luteipes (Scudder) (Dermaptera: Forficulidae) é um dos principais agentes de controle biológico natural de Spodoptera frugiperda (J. E. Smith) (Lepidoptera: Noctuidae), no Brasil, alimentando-se de ovos e lagartas pequenas em plantas de milho (Zea mays L.). Esse predador compartilha o mesmo habitat da praga, colocando seus ovos no interior do cartucho da planta. Dessa forma, aplicações de inseticidas para o controle desse inseto-praga podem reduzir a população do predador. O objetivo deste trabalho foi avaliar, em condições de semicampo, a seletividade de inseticidas para ninfas de primeiro e terceiro instares e adultos de $D$. luteipes. Os insetos foram colocados no interior dos cartuchos das plantas e receberam os inseticidas em pulverização com equipamento costal (pressão de 2,6 $\mathrm{lb} \mathrm{pol}^{-2}$, bico tipo quick Jet 8003 e 282 litros ha-1). Após a aplicação dos inseticidas, as plantas de milho contendo os insetos foram cobertas com gaiolas confeccionadas com arame e tecido. As avaliações foram feitas 24, 48 e 72 horas após a pulverização. Os inseticidas foram classificados de acordo com normas internacionais (IOBC). Para ninfas e adultos de D. luteipes, o inseticida etofenproxi (30 $\mathrm{g}$ i.a. ha $\left.{ }^{-1}\right)$ foi considerado levemente nocivo (classe 2); clorfenapir (180 g i.a. ha $\left.{ }^{-1}\right)$ foi moderadamente nocivo (classe 3) para ninfas e levemente nocivo (classe 2) para adultos; teflubenzurom/ $\alpha$-cipermetrina $\left(12,7 / 12,7 \mathrm{~g}\right.$ i.a. ha $\left.^{-1}\right)$ foi tóxico para ninfas de primeiro e terceiro instares 
(classe 4). Tiametoxam/ $\lambda$-cialotrina $\left(26,5 / 32,5 \mathrm{~g}\right.$ i.a. ha $\left.{ }^{-1}\right)$ foi considerado moderadamente nocivo para ninfas de terceiro instar e adultos, e para ninfas de primeiro instar nocivo. $\mathrm{O}$ inseticida espinosade (48 g i.a. ha- ${ }^{-1}$ ) foi moderadamente nocivo para ninfas de primeiro instar, levemente nocivo para as de terceiro instar e inócuo (classe 1) para os adultos. Triflumurom (24 g i.a. ha ${ }^{-1}$ ) foi levemente nocivo para ninfas e adultos de D. luteipes, o que o torna mais seletivo, visto que sua toxicidade é a mesma, independentemente da fase de desenvolvimento do predador.

Palavras-chave: controle biológico, lagarta-do-cartucho, predador, seletividade, tesourinhas.

\title{
EFFECT OF INSECTICIDES USED IN MAIZE (Zea mays L.) ON NYMPHS AND ADULTS OF Doru luteipes (SCUDDER) (DERMAPTERA: FORFICULIDAE) IN SEMI-FIELD CONDITION
}

\begin{abstract}
Doru luteipes (Scudder) (Dermaptera: Forficulidae) is one of the major agents of natural biological control of Spodoptera frugiperda (J. E. Smith) (Lepidoptera: Noctuidae) in Brazil, feeding on eggs and small larvae in maize (Zea mays L.) plants. This predator shares the same habitat of the pest, laying their eggs inside the whorl of the plant. Therefore, any application of insecticide to control the pest population affects the predator. The objective of this study was to evaluate the selectivity of insecticides on nymphs (first and third instars) and adults of D. luteipes, in a semi-field condition. Insects were placed inside the plant whorl and sprayed with backpack equipment (pressure $2.6 \mathrm{lb} \mathrm{in}^{-2}$, nozzle type quick Jet 8003 and 282 liters ha $^{-1}$ ). After the application of insecticides, maize plants were covered with cages made of wire frame and tissue. The assessments were done at 24, 48 and 72 hours after spraying. The insecticides were classified according to international standards (IOBC). The insecticides triflumuron (24 g a.i. ha $\left.{ }^{-1}\right)$ and etofenprox (30 g a.i. $\mathrm{ha}^{-1}$ ) were slightly harmful (class 2 ) to nymphs and adults of $D$. luteipes; the chlorfenapyr (180 g a.i. ha ${ }^{-1}$ ) was moderately harmful (class 3 ) for nymphs and slightly harmful (class 2) for adults; teflubenzuron/ $\alpha$-cypermethrin $\left(12.7 / 12.7 \mathrm{~g}\right.$ a.i. $\left.\mathrm{ha}^{-1}\right)$ was toxic to nymphs (class 4). Tiamethoxan/ $\lambda$-cyhalothrin $\left(26.5 / 32.5 \mathrm{~g}\right.$ a.i. ha $\left.{ }^{-1}\right)$ was moderately harmful to third instar nymphs and adults and harmful to first instar nymphs. The insecticide spinosad (48 g a.i. ha ${ }^{-1}$ ) was moderately harmful to first instar nymphs, slightly harmful to third instar nymphs and harmless to adults. Triflumuron (24 g a.i. ha-1) was slightly harmful to
\end{abstract}


nymphs and adults of $D$. luteipes, making it more selective since its toxicity is the same regardless of the developmental stage of the predator.

Key-words: biological control, fall armyworm, predator, selectivity, earwigs.

O milho (Zea mays L.) é um dos produtos agrícolas de ampla distribuição mundial, tanto na produção quanto no consumo, e tem-se caracterizado pela divisão da produção em duas épocas de plantio, no Brasil, o plantio de verão, ou primeira safra, e, mais recentemente, a produção da safra 2009/2010 obtida na safrinha, ou segunda safra. Somados os dois plantios, a produção foi em torno de 53,5 milhões de toneladas, o que o caracteriza como o segundo maior volume anual colhido no Brasil (Explosão..., 2010).

Vários são os fatores importantes e determinantes para a produção, destacandose, dentre eles, o clima, o manejo da cultura e principalmente os insetos-pragas. A ocorrência dos insetos-pragas e o seu controle fazem a diferença no rendimento final da cultura. Nesse contexto, a lagarta-do-cartucho, Spodoptera frugiperda(J.E.Smith)(Lepidoptera: Noctuidae) ocorre em todas as regiões produtoras, tanto no cultivo de verão quanto nos de segunda safra, sendo considerada a principal praga do milho, acarretando prejuízos de 400 milhões de dólares anualmente (Cruz, 1995; Cruz et al., 1999).

Essa praga, nos últimos anos, vem se destacando por sua severidade para a cultura do milho, em várias regiões brasileiras, devido, provavelmente, à eliminação de seus inimigos naturais e ao surgimento de populações resistentes a inseticidas, pois, logo que detectada na cultura, o seu controle tem sido realizado com produtos químicos. Atualmente há a opção do uso do milho Bt (Cruz et al., 2004), resistente às principais lagartas que atacam a parte aérea e as espigas da planta, como $S$. frugiperda, Diatraea saccharallis (Fabricius) (Lepidoptera: Pyralidae) e Helicoverpa zea (Boddie) (Lepidoptera: Noctuidae). No Brasil, o controle da $S$. frugiperda em lavoura de milho tem sido realizado com até 14 aplicações de inseticidas, sendo o controle biológico com inimigos naturais uma alternativa viável para o manejo desse noctuídeo (Valicente \& Tuelher, 2009). As "tesourinhas" Doru luteipes (Scudder) (Dermaptera: Forficulidae) são consideradas um dos mais importantes inimigos naturais de lagartas na cultura do milho. Ninfas e adultos são predadores vorazes de ovos e lagartas de primeiro instar de $S$. frugiperda e de $H$. zea (Cruz, 2009). O milho propicia alimentação e proteção para as fases jovens e adultas do predador, o qual pode ser encontrado em torno do $23^{\circ}$ dia após a emergência das plantas (Figueiredo et al., 2006) até a formação dos grãos nas espigas. A planta, além de proporcionar um habitat ideal para ovos e ninfas de D. luteipes, oferece também alimentação alternativa na forma de pólen. Os dermápteros 
são um dos primeiros predadores a serem observados nessa cultura, atuando sobre pragas primárias e secundárias (Guerreiro et al., 2003).

Por isso, o desenvolvimento de pesquisas com esse inimigo natural visando compatibilizar o uso do controle biológico com a aplicação de inseticidas em programas de manejo de pragas deve ser incentivado. De acordo com Hassan et al. (1985), pesquisadores membros da "International Organization for Biological and Integrated Control of Noxious Animals and Plants (IOBC), West Paleartic Regional Section (WPRS)", sugerem que deve-se incluir estudos da seletividade dos pesticidas sobre os inimigos naturais na adoção de estratégias de Manejo Integrado de Pragas (MIP).

Apesar da importância de $D$. luteipes como predador de $S$. frugiperda e da relevância do uso de inseticidas seletivos no manejo de pragas, raros trabalhos de pesquisa têm sido realizados na cultura do milho visando avaliar os efeitos tóxicos de compostos sobre essa espécie de predador, em condições de semicampo e campo. Assim, este trabalho teve como objetivo avaliar, em condições de semicampo, a seletividade de seis inseticidas recomendados para o controle da lagarta-do-cartucho do milho, para ninfas e adultos de D. luteipes.

\section{Material e Métodos}

O experimento foi realizado em novembro de 2010, em casa-de-vegetação, estabelecida na Embrapa Milho e Sorgo, em
Sete Lagoas, Minas Gerais, Brasil.

Adultos de D. luteipes presentes na cultivar de milho BRS 1030, conduzida em sistema orgânico, foram coletados e levados para o Laboratório de Criação de Insetos (LACRI) da Embrapa Milho e Sorgo, onde foram mantidos em sala climatizada a $25 \pm 2{ }^{\circ} \mathrm{C}$, umidade relativa de $70 \pm 10 \%$ e fotofase de 12 horas.

Casais de D. luteipes foram mantidos em gaiolas de criação confeccionadas com tubo PVC de $30 \mathrm{~cm}$ de diâmetro e $50 \mathrm{~cm}$ de altura. Suas extremidades foram fechadas com anéis de PVC de $2 \mathrm{~cm}$ de altura e tela de nylon com malha de $0,5 \mathrm{~mm}^{2}$. Dez cartuchos de milho foram colocados no interior, para que os insetos os utilizassem como abrigo e substrato para oviposição. Os insetos foram alimentados $a d$ libitum com ovos de S. frugiperda e dieta à base de ração de gato (Cruz, 2009).

A cultivar de milho BRS 1030 foi semeada na proporção de cinco sementes por vaso de plástico com capacidade para $5 \mathrm{~kg}$, tendo como substrato terra de barranco, previamente adubada com NPK 8-28-16. Após a emergência das plântulas, foi feito o desbaste, permanecendo duas plantas por vaso. Aos trinta dias de emergência, as plântulas foram infestadas com ninfas e adultos de $D$. luteipes, sendo que estes últimos estavam com três a quatro dias de idade. Em cada planta de milho, foram colocadas duas ninfas de primeiro e de terceiro instares e dois casais de adultos de D. luteipes, sendo que, juntamente com estes, foi introduzida uma postura de $S$. frugiperda tendo em média 
200 ovos como fonte alimentar dos predadores, durante o experimento. Após a infestação das plantas de milho com os insetos, foram realizadas as pulverizações com os diferentes inseticidas (Tabela 1), utilizando um pulverizador costal pressurizado a $\mathrm{CO}_{2}$, munido de um bico tipo quick Jet 8003, regulado à pressão de 2,6 lb poll- $^{-2}$, conferindo um volume de aplicação da ordem de 282 litros ha' ${ }^{-1}$, em jato dirigido.

Após as pulverizações, as plantas foram cobertas por gaiolas de arame galvanizado, com $18 \mathrm{~cm}$ de diâmetro e $50 \mathrm{~cm}$ de altura, revestidas com "voil". O delineamento experimental utilizado foi o de blocos casualizados, com sete tratamentos e cinco repetições, sendo que cada uma destas foi constituída por três vasos contendo duas plantas de milho. As avaliações foram realizadas 24, 48 e 72 horas após o início da exposição (HAIE) dos insetos aos inseticidas, considerando a mortalidade de ninfas e adultos.

Os dados coletados foram transformados para $(\mathrm{x}+0,5)^{1 / 2}$ e submetidos à análise de variância, sendo que as médias dos tratamentos foram comparadas entre si por meio do teste de agrupamento de Scott \& Knott, a 5\% de significância (Scott \& Knott, 1974), utilizando o programa Sisvar (Ferreira, 2007). Os dados de mortalidade dos insetos foram corrigidos pela fórmula de Abbott (Abbott, 1925) e os inseticidas classificados segundo índices de toxicidade propostos pela IOBC/WPRS, quando testados em semicampo (Hassan, 1997) em: 1 = inócuo $(<25 \%), 2=$ levemente nocivo $(25$ $50 \%), 3=$ moderadamente nocivo $(51-75 \%)$ e 4 $=$ nocivo $(>75 \%)$.

\section{Resultados e Discussão}

Não foram observadas diferenças significativas nas mortalidades de machos e fêmeas do predador provocadas pelos compostos avaliados $(\mathrm{P}>0,05)$. Espinosade apresentou $\mathrm{o}$ menor índice de mortalidade, que foi de $26,7 \%$ para os machos, sendo considerado levemente nocivo (classe 2) e inócuo para as fêmeas, cuja média foi de $25 \%$ (classe 1) às 72 HAIE (Tabela 2).

Os inseticidas triflumurom e etofemproxi mostraram-se levemente nocivos (classe 2), apesar de serem estatisticamente diferentes quanto à toxicidade para adultos, cujas médias

TABELA 1. Inseticidas utilizados nos bioensaios com D. luteipes.

\begin{tabular}{|c|c|c|c|c|c|}
\hline $\begin{array}{c}\text { Produto } \\
\text { comercial }\end{array}$ & Ingrediente ativo & Grupo químico & $\begin{array}{l}\text { Concentração } \\
\text {-Formulação }\end{array}$ & $\mathbf{D C}^{1}$ & D.i.a. ${ }^{2}$ \\
\hline Certero & triflumurom & Benzoilureia & $480-\mathrm{SC}$ & 0,05 & 24 \\
\hline Engeo Pleno & tiametoxam $/ \lambda$-cialotrina & Neonicotinoide/Piretroide & 141/106-SC & 0,25 & $26,5+32,5$ \\
\hline Imunit & teflubenzurom/ $\alpha$-cipermetrina & Piretroide/Benzoilureia & 75/75-SC & 0,17 & $12,7+12,7$ \\
\hline Pirate & clorfenapir & Análago de Pirazol & 240-SC & 0,75 & 180 \\
\hline Safety & etofemproxi & Éter Piretroide & 300-CE & 0,10 & 30 \\
\hline Tracer & espinosade & Espinosina & 480-SC & 0,10 & 48 \\
\hline
\end{tabular}


foram de 38 e $48 \%$ de mortalidade para os machos de D. luteipes e 38 e $45 \%$ para as fêmeas, respectivamente (Tabela 2).

Clorfenapir foi moderadamente nocivo (classe 3) para os machos e nocivo (classe 4) para as fêmeas, ocasionando mortalidade média de 66,7 e 78,3\%, respectivamente. Tiametoxam/ $\lambda$-cialotrina e teflubenzurom/ $\alpha$-cipermetrina às 72 HAIE causaram mortalidade de 96,7 e $95 \%$ para machos e fêmeas, respectivamente, sendo considerados nocivos (classe 4) (Tabela 2).

Para ninfas de primeiro e terceiro instares, nenhum dos produtos avaliados foi considerado inócuo (classe 1) 72 HAIE. Triflumurom foi levemente nocivo (classe 2) para ninfas de primeiro e terceiro instares, apresentando mortalidade média de $40 \%$ dos insetos. Tiametoxam $/ \lambda$-cialotrina e teflubenzurom $/ \alpha$ cipermetrina provocaram $100 \%$ de mortalidade de ninfas de primeiro instar às 72 HAIE, porém, para aquelas de terceiro instar, a mortalidade foi de 95 e 100\%, respectivamente, sendo também enquadrados como nocivos (classe 4). Clorfenapir, às 72 HAIE, causou $83,3 \%$ de mortalidade em ninfas de primeiro instar e de 95\% para as de terceiro instar, sendo também considerado nocivo ao predador (Tabela 3 ).

Etofemproxi, considerado moderadamente nocivo (classe 3), ocasionou mortalidades de 71,7 e $66,7 \%$ em contato com ninfas de primeiro e terceiro instares, respectivamente. Espinosade foi moderadamente nocivo (classe 3) para ninfas

TABELA 2. Mortalidade (\%) ( $\pm \mathrm{EP})$ de D. luteipes tratados diretamente com os inseticidas em diferentes intervalos de tempo e classes de toxicidade dos produtos.

\begin{tabular}{|c|c|c|c|c|}
\hline \multirow{2}{*}{ Tratamento } & \multicolumn{4}{|c|}{ Mortalidade de machos de $D$. luteipes $^{1}$} \\
\hline & $24 \mathrm{~h}$ & $48 \mathrm{~h}$ & $72 h$ & $\mathbf{C}^{2}$ \\
\hline Testemunha & $0,0 \pm 0,0 \mathrm{Ca}$ & $0,0 \pm 0,0 \mathrm{Ca}$ & $0,0 \pm 0,0 \mathrm{Ca}$ & - \\
\hline Triflumurom & $1,7 \pm 1,7 \mathrm{Cc}$ & $23,3 \pm 7,2 \mathrm{Cb}$ & $38,3 \pm 3,3 \mathrm{Ba}$ & 2 \\
\hline Tiametoxam $/ \lambda$-cialotrina & $83,3 \pm 5,3 \mathrm{Aa}$ & $96,7 \pm 3,3 \mathrm{Aa}$ & $96,7 \pm 3,3 \mathrm{Aa}$ & 4 \\
\hline Teflubenzurom/ $\alpha$-cipermetrina & $73,3 \pm 3,1 \mathrm{Aa}$ & $85,0 \pm 6,1 \mathrm{Aa}$ & $86,7 \pm 5,0 \mathrm{Aa}$ & 4 \\
\hline Clorfenapir & $60,0 \pm 11,0 \mathrm{Aa}$ & $66,7 \pm 8,8 \mathrm{Aa}$ & $66,7 \pm 8,8 \mathrm{Aa}$ & 3 \\
\hline Etofemproxi & $11,7 \pm 3,3 \mathrm{Bb}$ & $45,0 \pm 7,3 \mathrm{Ba}$ & $48,3 \pm 4,1 \mathrm{Ba}$ & 2 \\
\hline Espinosade & $10,0 \pm 3,1 \mathrm{Bb}$ & $21,7 \pm 6,3 \mathrm{Ca}$ & $26,7 \pm 6,7 \mathrm{Ca}$ & 2 \\
\hline $\mathrm{CV}(\%)$ & 23,9 & 21,0 & 15,1 & \\
\hline \multirow{2}{*}{ Tratamento } & \multicolumn{4}{|c|}{ Mortalidade de fêmeas de $D$. luteipes $^{1}$} \\
\hline & $24 \mathrm{~h}$ & $48 \mathrm{~h}$ & $72 \mathrm{~h}$ & $\mathbf{C}^{2}$ \\
\hline Testemunha & $0,0 \pm 0,0 \mathrm{Ca}$ & $0,0 \pm 0,0 \mathrm{Ca}$ & $0,0 \pm 0,0 \mathrm{Ca}$ & - \\
\hline Triflumurom & $3,30 \pm 2,0 \mathrm{Cc}$ & $15,0 \pm 3,1 \mathrm{Cb}$ & $38,30 \pm 6,8 \mathrm{Ba}$ & 2 \\
\hline Tiametoxam $/ \lambda$-cialotrina & $88,3 \pm 8,2 \mathrm{Aa}$ & $98,3 \pm 1,7 \mathrm{Aa}$ & $100 \pm 0,00 \mathrm{Aa}$ & 4 \\
\hline Teflubenzurom/ $\alpha$-cipermetrina & $83,3 \pm 5,9 \mathrm{Aa}$ & $95,0 \pm 5,0 \mathrm{Aa}$ & $95,0 \pm 5,0 \mathrm{Aa}$ & 4 \\
\hline Clorfenapir & $71,7 \pm 8,2 \mathrm{Aa}$ & $78,3 \pm 6,8 \mathrm{Aa}$ & $78,30 \pm 6,8 \mathrm{Aa}$ & 4 \\
\hline Etofemproxi & $21,7 \pm 3,3 \mathrm{Bb}$ & $43,0 \pm 3,1 \mathrm{Ba}$ & $45,0 \pm 3,30 \mathrm{Ba}$ & 2 \\
\hline Espinosade & $16,7 \pm 7,9 \mathrm{Ba}$ & $23,3 \pm 8,9 \mathrm{Ca}$ & $25,0 \pm 9,5 \mathrm{Ca}$ & 1 \\
\hline $\mathrm{CV}(\%)$ & 21,2 & 16,0 & 16,2 & \\
\hline
\end{tabular}


de primeiro instar, cuja mortalidade média foi de $61,7 \%$ e levemente nocivo (classe 2 ) para as ninfas de terceiro instar, ocasionando a morte de 46,7\% (Tabela 3).

O inseticida fisiológico triflumurom, pertencente ao grupo das benzoilureias, atua por contato ou ingestão, inibindo a formação de quitina, interferindo no processo de esclerotização da cutícula (Guedes, 1999). Segundo Cruz (1993), uma alternativa ecológica em programas de manejo integrado de $S$. frugiperda, na cultura do milho, seria o uso de inseticidas inibidores da síntese de quitina, os quais apresentam baixa toxicidade aos mamíferos e aos inimigos naturais, além de baixa contaminação no ambiente.
Os resultados deste trabalho divergem em parte daqueles obtidos por Simões et al. (1998), que, ao avaliarem a seletividade de triflumurom (Alsystin 250 PM - $100 \mathrm{~g} \mathrm{ha}^{-1}$ ) para D. luteipes, em diferentes fases de desenvolvimento, constataram que a resposta do inseto ao composto variou em função dos diferentes instares testados e que os adultos do predador foram mais tolerantes. Triflumurom ocasionou a mortalidade de 77,1, 66,4 e $47,7 \%$ dos insetos de primeiro e terceiro instares e adultos, respectivamente. No entanto, valores inferiores foram observados, sendo que as médias de mortalidade ocasionadas pelo triflumurom foram de 38,3\% para adultos de $D$. luteipes e de $40 \%$ para ninfas, sendo considerado levemente nocivo (classe 2).

TABELA3. Mortalidade ( $\%)( \pm \mathrm{EP})$ de ninfas de $D$. luteipes de primeiro e terceiro instares diretamente tratadas com os inseticidas em diferentes intervalos de tempo e classificação de toxicidade dos produtos.

\begin{tabular}{|c|c|c|c|c|}
\hline \multirow{2}{*}{ Tratamento } & \multicolumn{4}{|c|}{ Ninfas de primeiro instar de $D$. luteipes $^{1}$} \\
\hline & 24h & $48 \mathrm{~h}$ & $72 \mathrm{~h}$ & $\mathbf{C}^{2}$ \\
\hline Testemunha & $0,0 \pm 0,0 \mathrm{Da}$ & $0,0 \pm 0,0 \mathrm{Da}$ & $0,0 \pm 0,0 \mathrm{Da}$ & - \\
\hline Triflumurom & $10,0 \pm 4,9 \mathrm{Db}$ & $33,3 \pm 0,46 \mathrm{Ca}$ & $40,0 \pm 4,1 \mathrm{Ca}$ & 2 \\
\hline Tiametoxam $/ \lambda$-cialotrina & $93,3 \pm 3,1 \mathrm{Aa}$ & $95,0 \pm 2,0 \mathrm{Aa}$ & $100 \pm 0,00 \mathrm{a}$ & 4 \\
\hline Teflubenzurom $/ \alpha$-cipermetrina & $93,3 \pm 3,1 \mathrm{Aa}$ & $96,7 \pm 3,3 \mathrm{Aa}$ & $100 \pm 0,0 \mathrm{Aa}$ & 4 \\
\hline Clorfenapir & $80,0 \pm 8,6 \mathrm{Aa}$ & $83,3 \pm 7,9 \mathrm{Aa}$ & $83,3 \pm 7,9 \mathrm{Aa}$ & 4 \\
\hline Etofemproxi & $50,0 \pm 5,9 \mathrm{Ba}$ & $66,7 \pm 3,7 \mathrm{Ba}$ & $71,7 \pm 5,7 \mathrm{Ba}$ & 3 \\
\hline Espinosade & $38,3 \pm 10,4 \mathrm{Ca}$ & $58,3 \pm 14,7 \mathrm{Ba}$ & $61,7 \pm 15,5 \mathrm{Ba}$ & 3 \\
\hline CV (\%) & 21,4 & 19,4 & 16,9 & \\
\hline \multirow{2}{*}{ Tratamento } & \multicolumn{4}{|c|}{ Ninfas de terceiro instar de $D$. luteipes ${ }^{1}$} \\
\hline & $24 \mathrm{~h}$ & $48 \mathrm{~h}$ & $72 \mathrm{~h}$ & $\mathbf{C}^{2}$ \\
\hline Testemunha & $0,0 \pm 0,0 \mathrm{Da}$ & $0,0 \pm 0,0 \mathrm{Da}$ & $0,0 \pm 0,0 \mathrm{Da}$ & - \\
\hline Triflumurom & $3,30 \pm 2,0 \mathrm{Db}$ & $26,7 \pm 5,5 \mathrm{Ca}$ & $40,0 \pm 9,7 \mathrm{Ca}$ & 2 \\
\hline Tiametoxam/ $\lambda$-cialotrina & $80,0 \pm 8,6 \mathrm{Aa}$ & $91,7 \pm 4,6 \mathrm{Aa}$ & $95,0 \pm 3,3 \mathrm{Aa}$ & 4 \\
\hline Teflubenzurom/ $\alpha$-cipermetrina & $81,7 \pm 1,7 \mathrm{Aa}$ & $100 \pm 0,0 \mathrm{Aa}$ & - & 4 \\
\hline Clorfenapir & $71,7 \pm 3,3 \mathrm{Ba}$ & $95,0 \pm 3,3 \mathrm{Aa}$ & $95,0 \pm 3,3 \mathrm{Aa}$ & 4 \\
\hline Etofemproxi & $50,0 \pm 12,7 \mathrm{Ba}$ & $63,3 \pm 7,3 \mathrm{Ba}$ & $66,7 \pm 8,4 \mathrm{Ba}$ & 3 \\
\hline Espinosade & $26,7 \pm 7,2 \mathrm{Ca}$ & $43,3 \pm 13,6 \mathrm{Ca}$ & $46,7 \pm 15,1 \mathrm{Ca}$ & 2 \\
\hline CV $(\%)$ & 20,6 & 16,6 & 16,5 & \\
\hline
\end{tabular}

${ }^{2}$ Classe de toxicidade segundo IOBC/WPRS (Hassan, 1997). 
Zotti et al. (2008) avaliaram a seletividade dos principais inseticidas utilizados na cultura do milho para ninfas de primeiro instar e adultos de Doru lineare (Eschscholtz) (Dermaptera: Forficulidae), em condições de semicampo. Baseando-se na mortalidade dos insetos às 72 horas após a sua exposição aos compostos, constataram que triflumurom foi inócuo para ninfas (classe 1), propiciando mortalidade abaixo de $25 \%$. No entanto, esses resultados divergem para adultos de $D$. luteipes de ambos os sexos e para suas ninfas de primeiro e terceiro instares, em que triflumurom foi considerado levemente nocivo (classe 2), ocasionando mortalidade superior a $25 \%$.

Adultos de $D$. lineare, após serem expostos a deltametrina, $\alpha$-cipermetrina, lufenurom e azadiraquitina, apresentaram mortalidade abaixo de $25 \%$, sendo considerados inócuos, porém o inseticida diflubenzurom foi levemente nocivo (classe 2) ao predador na fase adulta (Zotti et al., 2008). Isso indica uma toxicidade diferenciada quanto ao efeito de deltametrina sobre $D$. lineare e $D$. luteipes. Conforme resultados de Simões et al. (1998), deltametrina (Decis 25 CE - $150 \mathrm{ml} \mathrm{ha}^{-1}$ ) apresentou menor efeito sobre ninfas de $D$. luteipes, com índices de mortalidade média de 17,7 e $26,5 \%$ para os insetos de primeiro e terceiro instares, respectivamente, e de $47,7 \%$ para os adultos do predador.

Quanto ao inseticida $\lambda$-cialotrina (Karate 50 CE $150 \mathrm{ml} \mathrm{ha}^{-1}$ ), Simões et al. (1998) comprovaram a mortalidade de $100 \%$ das ninfas de $D$. luteipes. Resultado semelhante foi observado para ninfas de primeiro e terceiro instares no presente estudo. Para as de primeiro instar, ocorreu $100 \%$ de mortalidade às 72 HAIE, enquanto que, para as de terceiro instar, verificou-se $100 \%$ de mortalidade às 48 HAIE, sendo o composto enquadrado na classe 4 . Em ambos os trabalhos, nota-se que ninfas e adultos de $D$. luteipes foram bastante afetados pela mistura tiametoxam $/ \lambda$-cialotrina, como também em aplicações de apenas $\lambda$-cialotrina.

Resultados divergentes foram observados por Zotti et al. (2008), pois o composto $\lambda$-cialotrina foi considerado levemente nocivo (classe 2) para $D$. lineare, porém deve ser levado em conta que, neste trabalho, foram testados os efeitos da mistura tiametoxam $/ \lambda$-cialotrina sobre D. luteipes, cuja mortalidade foi alta em ambos os estádios, e nesse caso, considerada nocivo.

Simas et al. (2006), com objetivo de avaliar em campo a seletividade de tiametoxam $+\lambda$-cialotrina para $D$. lineare e Chrysoperla sp. (Neuroptera: Chrysopidae), em cultivo de soja, concluíram que a mistura inseticida foi altamente tóxica, reduzindo em mais de $90 \%$ as populações desses predadores após a sua aplicação. Esse mesmo produto apresentou resultados semelhantes para ninfas de primeiro e terceiro instares, assim como para os adultos de $D$. luteipes, cuja mortalidade foi superior a $95 \%$, sendo considerado nocivo (classe 4).

Os piretroides atuam por contato e ingestão, sendo sua atuação nos canais de sódio, posicionando-se em alguns sítios de ligação 
de tal modo que estes permanecem abertos por um tempo maior, prolongando-se, assim, o período de influxo de sódio, e, com isso, os insetos morrem, devido à hiperexcitabilidade provocada. Já os compostos neurotóxicos, como os neonicotinoides, atuam como agonistas da acetilcolina, ligando-se aos receptores nicotínicos, abrindo os canais de sódio; entretanto, as moléculas não são degradadas imediatamente, levando à hiperexcitação do sistema nervoso e, consequentemente, à morte dos insetos (Omoto, 2000).

Os inseticidas formados de dois princípios ativos, teflubenzurom/ $\alpha$-cipermetrina e tiametoxam/ $\lambda$-cialotrina, foram nocivos (classe 4 ) para ninfas de primeiro e terceiro instares, assim como para os machos e fêmeas de D. luteipes.

Alta mortalidade ocasionada por teflubenzurom $/ \alpha$-cipermetrina e tiametoxam $/ \lambda$ cialotrina provavelmente se deve à associação de dois princípios ativos (piretroide + benzoilureia e neonicotinoide + piretroide), respectivamente, cujos mecanismos possuem ação diferente, agindo simultaneamente sobre um mesmo inseto, podendo aumentar consideravelmente a sua toxicidade (Rigitano \& Carvalho, 2001).

Espinosade apresentou efeito diferenciado para D. luteipes, sendo considerado classe 1 para as fêmeas, classe 2 para machos e ninfas de terceiro instar e classe 3 para ninfas de primeiro instar, enquanto Zotti et al. (2008) observaram que esse inseticida se mostrou moderadamente nocivo (classe 3 ) para ninfas de $D$. lineare.

Espinosade foi inócuo (classe 1) para fêmeas e levemente nocivo para os machos e ninfas de terceiro instar (classe 2), porém foi moderadamente nocivo a ninfas de primeiro instar. Esse inseticida teve ação diferenciada sobre o predador e, apesar disso, também pode ser recomendado em programas de manejo de $S$. frugiperda, visando a sua preservação.

A baixa toxicidade do espinosade para $D$. luteipes pode ser devido ao fato de o inseticida ser particularmente ativo contra lepidópteros e dípteros, agindo por contato e, principalmente, por ingestão. É uma neurotoxina que atua como agonista da acetilcolina, pertencente ao grupo químico das espinosinas, capaz de se ligar aos receptores nicotínicos desse neurotransmissor, impedindo a sua degradação pela enzima acetilcolinesterase nas sinapses. Dessa forma, a exposição dos insetos a esse composto resulta em baixa alimentação, seguida de paralisia e morte (Salgado et al., 1998).

A seletividade de tiametoxam e etofemproxi para predadores das pragas do algodoeiro, em campo, foi estudada por Lima Junior et al. (2010). Os inseticidas foram aplicados 84 dias após a emergência do algodoeiro e as avaliações realizadas antes e até 33 dias após a aplicação dos inseticidas. Segundo os autores, foram observados os inimigos naturais Cycloneda sanguinea (Linnaeus); Hippodamia convergens GuérinMéneville; Eriopis conexa (Germar); Scymnus spp. (Coleoptera: Coccinellidae); Geocoris spp. (Hemiptera: Geocoridae); Zellus spp. (Hemiptera: Reduviidae); Podisus 
spp. (Hemiptera: Pentatomidae) e Doru sp. (Dermaptera: Forficulidae). De acordo com as observações, tiametoxam não foi seletivo ao complexo de predadores ocorrentes, com porcentagens de mortalidade que oscilaram entre 27,5 e $72 \%$, sendo, assim, classificado no final das avaliações como moderadamente nocivo. Foi observado para D. luteipes que o mesmo produto, quando associado a $\lambda$-cialotrina, apresentou mortalidade maior que $75 \%$, mudando sua classificação de moderadamente nocivo para nocivo (classe 4).

Segundo Lima Junior et al. (2010), etofemproxi apresentou mortalidade de $86 \%$ no terceiro dia de avaliação, sendo considerado como nocivo a Doru sp. e aos principais inimigos naturais presentes no algodoeiro, resultados esses divergentes dos observados para ninfas de primeiro e terceiro instares de $D$. luteipes, onde foi considerado moderadamente nocivo (classe 3); porém, para os adultos, esse inseticida foi menos tóxico e classificado como levemente nocivo (classe 2).

Clorfenapirfoi consideradomoderadamente nocivo aos machos de D. luteipes (classe 3), porém as fềmeas e ninfas de primeiro e terceiro instares foram mais sensíveis ao inseticida, sendo, assim, classificado como nocivo (classe 4).

A menor tolerância das ninfas de $D$. luteipes aos produtos testados assemelhase aos resultados obtidos nos trabalhos de Faleiro et al. (1995), Simões et al. (1998) e Picanço et al. (2003), nos quais foi observado que vários inimigos naturais quando recém- nascidos e ou mais jovens, apresentaram maior susceptibilidade a moléculas químicas, geralmente por apresentarem baixa capacidade de metabolização dos compostos, o que provavelmente dificultou a sua desintoxicação. A menor espessura do exoesqueleto nesse estágio de desenvolvimento também pode facilitar a penetração dos inseticidas na cutícula do inseto (Hollingwort, 1976).

Alguns resultados de pesquisas a respeito da toxicidade de inseticidas para predadores, principalmente dermápteros, corroboram os obtidos neste trabalho, confirmando a maior tolerância dos adultos de D. luteipes aos inseticidas, quando comparados com a fase jovem.

É importante salientar que os inseticidas que foram enquadrados nas classes 3 e 4 de toxicidade para ninfas e/ou adultos de $D$. luteipes, devem ser avaliados em condições de campo, uma vez que, nessas condições, o predador terá chances de evitar o contato com os produtos, migrando do local tratado ou se abrigando naqueles não tratados. Em condições de campo, os inimigos naturais geralmente ficam menos expostos aos resíduos dos inseticidas, em função dos refúgios existentes nas plantas. É possível que uma planta de milho em estádio avançado de desenvolvimento possa ser utilizada como meio de escape e/ou refúgio dos inimigos naturais aos efeitos dos inseticidas. Formulações consideradas muito tóxicas em laboratório podem ter uma ação menos tóxica nessas condições (Pedigo, 1999; Zotti et al., 2008). 
Tiametoxam/ $\lambda$-cialotrina, teflubenzurom/ $\alpha$-cipermetrina e clorfenapir devem ser evitados em lavouras onde se encontram D. luteipes, devido à sua alta toxicidade sobre ninfas e adultos deste predador.

Com restrições, etofenproxi e espinosade podem ser aplicados, porém deve-se evitar as pulverizações quando observada maior densidade de ninfas nas plantas, pois estas foram mais sensíveis ao produto do que os adultos de $D$. luteipes.

Triflumurom foi considerado levemente nocivo para ninfas e adultos de D. luteipes; logo, seria o produto mais seletivo e de menor impacto sobre este inimigo natural no campo, visto que esses resultados foram obtidos em local semiconfinado e, provavelmente, no agroecossistema, os resultados poderiam vir a ser mais favoráveis.

\section{Agradecimentos}

Ao Conselho Nacional de Desenvolvimento Científico e Tecnológico (CNPq), à Fundação de Amparo à Pesquisa do Estado de Minas Gerais (FAPEMIG) e à Coordenação de Aperfeiçoamento de Pessoal de Nível Superior (CAPES), o apoio financeiro.

\section{Literatura Citada}

ABBOTT, W. S. A method of computing the effectiveness of an insecticide. Journal of Economic Entomology, College Park, v. 18, p. 265267, 1925.
CRUZ, I. Aplicação de inseticidas para o controle da lagarta-do-cartucho, Spodoptera frugiperda, e sua ação sobre o inimigo natural Doru luteipes. Relatório Técnico Anual do Centro Nacional de Pesquisa de Milho e Sorgo 1991-1993, Sete Lagoas, v. 6, p. 82, 1993.

CRUZ, I. Manejo integrado de pragas de milho com ênfase para o controle biológico. Anais da Sociedade Entomológica do Brasil, Jaboticabal, p. 48-92, 1995.

CRUZ. I. Métodos e criação de agentes entomófogos de Spodoptera frugiperda. In: BUENO, V. H. P. (Ed.). Controle biológico de pragas: produção massal e controle de qualidade. Lavras: UFLA, 2009. v. 2, p. 237-248.

CRUZ, I.; FIGUEIREDO, M. L. C.; OLIVEIRA, A. C.; VASCONSELOS, C. A. Damage of Spodoptera frugiperda (Smith) in different maize genotypes cultivated in soil under three levels of aluminium saturation. International Journal of Pest Management, London, v. 45, p. 293-296, 1999.

CRUZ, I.; CUNHA, J. R.; FIGUEIREDO, M. L. C. Avaliação de diferentes doses do inseticida Akito (betacypermetrina) sobre larvas de Spodoptera frugiperda e sobre os predadores Doru luteipes e Chrysoperla externa. In: CONGRESSO NACIONAL DE MILHO E SORGO, 25.; SIMPÓSIO BRASILEIRO SOBRE A LAGARTA-DO-CARTUCHO, SPODOPTERA FRUGIPERDA, 1., 2004, Cuiabá. Resumos... Sete Lagoas: ABMS: Embrapa Milho e Sorgo; Cuiaba; Empaer, 2004. p. 133.

EXPLOSÃO produtiva: plantio na safrinha bate recordes... Anuário Brasileiro do Milho, Santa Cruz do Sul, p. 12-14, 2010.

FALEIRO, F. G.; PICANÇO, M. C.; PAULA, S. V.; BATALHA, V. C. Seletividade de inseticidas a Spodoptera frugiperda (J. E. Smith) (Lepidoptera: 
Noctuidae) e ao predador Doru luteipes (Scudder) (Dermaptera: Forficulidae). Anais da Sociedade Entomológica do Brasil, Jaboticabal, v. 24, p. 247252, 1995.

FERREIRA, D. F. SISVAR: programa estatístico: versão 5.0. Lavras: UFLA, 2007. Software.

FIGUEIREDO, M. L. C., PENTEADO-DIAS, A. M., CRUZ, I. Efeito do inseticida chlorpyrifos e sua interação com inimigos naturais na supressão de Spodoptera frugiperda (J. E. Smith, 1797) (Lepidoptera: Noctuidae) na cultura do milho. Revista Brasileira de Milho e Sorgo, Sete Lagoas, v. 5, n. 3, p. 325-339, 2006.

GUEDES, R. N. C. Mecanismos de ação de inseticidas. In: OMOTO, C.; GUEDES, R. N. C. Resistência de pragas a pesticidas: princípios e práticas. Mogi Mirim: IRAC-BR, 1999. p. 24-26.

GUERREIRO, J. C.; BERTI FILHO, E.; BUSOLI, A. C. Ocorrência estacional de Doru luteipes na cultura do milho em São Paulo, Brasil. Manejo Integrado de Plagas y Agroecología, Costa Rica, n. 70, p. 46-49, 2003.

HASSAN, S. A.; BIGLER, F.; BLAISINGER, P.; BOGENSCHÜTZ, H.; BRUN, J.; CHIVERTON, P.;DICKLER, E.; EASTERBROOK, M. A.; EDWARDS, P. J.; ENGLERT, W. D.; FIRTH, S. I.; HUANG, P.; INGLESFIELD, D.; KLINGAUF, F.; KÜHNER, C.; LEDIEU, M. S.; NATON, E.; OOMEN, P. A.; SHIRES, S. W.; STÄUBLI, A.; STEVENSON, J.; TUSET, J. J.; VANWETSWINKEL, G.; van ZON,A.Q. P. Standard methods to test the side effects of pesticides on natural enemies of insects and mitesdevelopped by the IOBC/WPRS working group "Pesticides and beneficial organisms". OEPP/EPPO Bulletin, Oxford, v. 15, p. 214-255, 1985.

HASSAN, S. A. Métodos padronizados para testes de seletividade com ênfase em Trichogramma. In: PARRA, J. R. P.; ZUCCHI, R. A. (Ed.). Trichogramma e o controle biológico aplicado. Piracicaba: FEALQ, 1997. cap. 8, p. 207-233.

HOLLINGWORTH, R. M. The biochemical and physiological basis of selective toxicity. In: WILKINSON, C. F. (Ed.). Insecticide biochemistry and physiology. New York: Plenum, 1976. p. 431-506.

LIMA JUNIOR, I. S.; NOGUEIRA, R. F.; BERTONCELLO, T. F.; MELO, E. P.; SUEKANE, R.; DEGRANDE, P. E. Seletividade de inseticidas sobre o complexo de predadores das pragas do algodoeiro. Pesquisa Agropecuária Tropical, Goiânia, v. 40, n. 3, p. 347-353, 2010.

OMOTO, C. Modo de ação de inseticidas e resistência de insetos a inseticidas. In: GUEDES, J. C.; COSTA, I. D.; CASTIGLIONI, E. (Org.) Bases e técnicas do manejo de insetos. Santa Maria: Pallotti, 2000. p. 31-49.

PEDIGO, L. P. Entomology and pest management. 3. ed. Englewood: Prentice Hall, 1999. 691 p.

PICANÇO, M. C.; MOURA'M. F.; MIRANDA, M. M. M.; GONTIJO, L. M.; FERNANDES, F. L. Seletividade de inseticidas a Doru luteipes (Scudder, 1876) (Dermaptera: Forficulidae) e Cotesia sp. (Hymenoptera: Braconidae) inimigos naturais de Ascia monuste orseis (Godart, 1818) (Lepidoptera: Pieridae). Ciência Rural, Santa Maria, v. 33, n. 2, p. 183-188, 2003.

RIGITANO, R. L. O; CARVAlHO, G. A. Toxicologia e seletividade de inseticidas. Lavras: UFLA: FAEPE, 2001. 72 p.

SALGADO, V. L.; SHEETS, J. J.; WATSON, G. B.; SCHMIDT, A. L. Studies on the mode of action of spinosad: insect symptoms and physiological 
correlates. Pesticide Biochemistry and Physiology, New York, v. 60, p. 103-110, 1998.

SCOTT, A. J.; KNOTT, M. A. A cluster analyses methodforgroupingmeansintheanalysesofvariance. Biometrics, Washington, v. 30, p. 507-512, 1974.

SIMAS, J. R.; FRANÇA, J. A. S.; SULZBACH, F.; BIGOLIN, M.; FIORIN, R. A.; MAZIERO, H.; GUEDES, J. V. C. Eficiência de tiametoxam + lambda-cialotrina no controle do percevejoverde-pequeno, Piezodorus guildini (Westwood, 1837) (Hemiptera: Pentatomidae) e seletividade para predadores na cultura da soja. Revista da Faculdade de Zootecnia, Veterinária e Agronomia, Uruguaiana, v. 13, n. 2, p. 10-19, 2006.

SIMÕES, J. C.; CRUZ, I.; SALGADO, L. O. Seletividade de inseticidas às diferentes fases de desenvolvimento do predador Doru luteipes (Scudder) (Dermaptera: Forficulidae). Anais da Sociedade Entomológica do Brasil, Jaboticabal, v. 27, n. 2, p. 289-294, 1998.

VALICENTE, F. H.; TUELHER, E. de S. Controle biológico da lagarta do cartucho, Spodoptera frugiperda, com baculovírus. Sete Lagoas: Embrapa Milho e Sorgo, 2009. 14 p. (Embrapa Milho e Sorgo. Circular técnica, 114).

ZOTTI, M. J. ; GRÜTZMACHER, A. D.; GRUTZMACHER, D. D.; DALMAZO, G. O.; MARTINS, J. F. S. Seletividade de inseticidas usados na cultura do milho para ninfas e adultos do predador Doru lineare (Eschcholtz, 1822) (Dermaptera: Forficulidae) em condições de semicampo. Revista Brasileira de Agrociência, Pelotas, v. 14, n. 3, 2008. 